\title{
POUR UNE VISION PÉRIPHÉRIQUE DE LA SÉMANTIQUE LINGUISTIQUE PRÉSENTE ET À VENIR
}

Jacques François, Dirk Geeraerts

Armand Colin | « Langages »

2016/1 N²01 | pages 5 à 14

ISSN 0458-726X

ISBN 9782200930394

Article disponible en ligne à l'adresse :

http://www.cairn.info/revue-langages-2016-1-page-5.htm

\section{Pour citer cet article :}

Jacques François, Dirk Geeraerts, « Pour une vision périphérique de la sémantique linguistique présente et à venir », Langages 2016/1 (N²01), p. 5-14.

DOI 10.3917/lang.201.0005

Distribution électronique Cairn.info pour Armand Colin.

(C) Armand Colin. Tous droits réservés pour tous pays.

La reproduction ou représentation de cet article, notamment par photocopie, n'est autorisée que dans les limites des conditions générales d'utilisation du site ou, le cas échéant, des conditions générales de la licence souscrite par votre établissement. Toute autre reproduction ou représentation, en tout ou partie, sous quelque forme et de quelque manière que ce soit, est interdite sauf accord préalable et écrit de l'éditeur, en dehors des cas prévus par la législation en vigueur en France. Il est précisé que son stockage dans une base de données est également interdit. 


\section{Jacques François}

Université de Caen \& Laboratoire Lattice (UMR 8094 CNRS - ENS - Université Sorbonne Nouvelle-Paris 3)

Dirk Geeraerts

Katholieke Universiteit Leuven

\section{Pour une vision périphérique de la sémantique linguistique présente et à venir}

F. de Saussure - ou en tout cas le Saussure de la tradition structuraliste européenne - nous a légué une vision « autonomiste » de la sémantique linguistique qui a permis le développement de la théorie des champs sémantiques en synchronie (cf. Weisgerber 1929 et la Sprachinhaltsforschung ${ }^{1}$ ) et de leur évolution en diachronie (cf. Trier 1931), la théorisation de la sémantique componentielle (Hjelmslev 1943 ; Coseriu 1958 ; Weisgerber 1962 ; Wierzbicka 1972) et son application à l'anthropologie linguistique (Goodenough 1956 ; Loundsbury 1964) et à la narratologie (Barthes 1964 ; Greimas 1965) et la clarification du jeu complexe des deux dimensions onomasiologique (« des idées vers les mots ») et sémasiologique ( des mots vers les idées »), spécialement en Allemagne (cf. Baldinger 1984 pour une synthèse). Le bilan est donc très honorable. Cependant, cette vision autonomiste a son revers de la médaille (sans allusion excessive à la métaphore saussurienne des deux faces d'un tissu) :

- d'une part, elle est implicitement héritière de la vision humboldtienne de la «forme interne de la langue » (innere Form der Sprache) et est donc en phase avec l'hypothèse relativiste représentée par la tradition « culturaliste ${ }^{2}$; de ce fait, la question de L'ACCESSIBILITÉ ENTRE DEUX «SYSTÈMES SÉMANTIQUES » est éludée : toute conceptualisation est le fruit d'un système d'oppositions sémantiques particulier à une langue,

- et même sur le plan intralinguistique, l'idée de l'homogénéité du système linguistique chère aux structuralistes a pu induire un certain désintérêt à l'égard de la variation sémantique ;

- d'autre part, la question de la RÉFÉRENCE, et donc celle du jeu des désignations alternatives pour un référent, constitue un point aveugle de cette conception ;

1. Litt. 'Recherche sur le contenu de la langue'.

2. Voir la contribution de Everett (2015, ce numéro) et Geeraerts (2010, chap. 3 « Structuralist semantics »). 
- enfin, du fait que, pour F. de Saussure, la syntaxe relève de la parole, et non de la langue, la question de la COMPOSITION DU SENS est elle aussi hors de propos.

C'est ce qui explique que - malgré l'entreprise de F. Rastier pour réhabiliter la sémantique autonome de F. de Saussure et, plus largement, du structuralisme européen (avec Hjelmslev et Coseriu en fers de lance ; cf. Hjelmslev 1997, Coseriu 2001) - la sémantique linguistique pratiquée actuellement s'inspire :

- fréquemment du second L. Wittgenstein, celui des Investigations philosophiques (1961) et, dans son prolongement, de la théorie de la pragmatique intégrée (Ducrot 1989), de la pertinence (cf. Moeschler 1989) et de la polyphonie (Bres et al. 2005) ;

- ou, occasionnellement, de la sémantique cognitive américaine et, en particulier, de la théorie de la cognition incarnée (cf. Lakoff \& Johnson 1999 ; Langacker 2008) ;

- ou, plus marginalement, dans l'optique de la corrélation entre structures sémantiques et syntaxiques, des entreprises de formalisation (cf. Dowty 1979 ; Kamp \& Reyle 1993).

Mais au-delà - ou plutôt en-deça - de ces cadres éminemment théoriques, la sémantique linguistique en France, et notamment celle qui traite de la description sémantique du français telle qu'elle est illustrée par les projets de communications soumises à de grands congrès ${ }^{3}$, donne souvent l'impression d'une démarche centrée sur des questions extrêmement limitées, quasiment microscopiques, avec un engagement théorique limité, voire minimal, comme si l'inscription d'une étude particulière dans un champ théorique balisé depuis des décennies et la prise en compte des observations effectuées sur l'organisation sémantique d'autres langues et sur la comparaison entre différentes langues de culture voisine ou éloignée étaient d'un intérêt secondaire, marginal, voire négligeable. Il va sans dire que cette critique ne touche pas l'ensemble des projets de communication en cause, mais cependant une partie, non négligeable, de ces projets. La sémantique linguistique semble y devenir « autonome » dans un sens nouveau et quelque peu préoccupant : par réduction à un micro-questionnement et mise entre parenthèses d'une problématique sémantique plus vaste, impliquant des interrogations telles que :

- est-ce que je conçois l'examen d'une question sémantique en synchronie comme auto-suffisant ? Sinon, quelle est ma conception de la place des usages actuellement dominants, entre les usages archaïques et les usages innovants ?

\footnotetext{
3. Ce jugement se fonde sur l'évaluation demandée à Jacques François de centaines de projets de communication dans la section «Sémantique » de trois manifestations linguistiques de grande ampleur : les deux premiers Congrès Mondiaux de Linguistique Française (2008 à Paris, 2010 à La Nouvelle-Orléans) et le $27^{e}$ Congrès de la Société de Linguistique Romane (2013 à Nancy).
} 
- est-ce que je me situe plutôt du côté d'une sémantique dénuée de perspective référentielle ou d'une sémantique exclusivement référentielle, ou est-ce que je cherche ma voie entre les deux?

- est-ce que je conçois la question sémantique que je traite comme indépendante des conditions d'énonciation ou comme un objet d'étude "pragmasémantique »?

- suis-je en droit de m'imputer, en tant que locuteur natif, une capacité de jugement suffisante pour identifier le jeu des relations de sens dans ma langue, ou dois-je faire confiance à la tradition des lexicographes (particulièrement riche pour le français), ou encore pratiquer l'analyse de corpus textuels afin $\mathrm{d}$ 'attribuer une place particulière à mon propre usage par rapport à celui de la communauté linguistique à laquelle j'appartiens ?

- suis-je d'avis qu'en savoir plus sur le traitement du sens linguistique dans la communication, dans l'acquisition de la langue maternelle ou d'une langue seconde, sur les pathologies perturbant son traitement et sur la manière dont l'espèce humaine est supposée avoir commencé à échanger du sens par la parole, peut m'apporter un éclairage propre à élargir ou réorienter ma propre investigation?

Le présent numéro ne se propose pas de fournir des recommandations, il énumère des éclairages variés qui reflètent chacun une approche plus ou moins périphérique de la sémantique linguistique. Que faut-il entendre par " périphérique " et par "plus ou moins » ? Les huit articles qui suivent sont autant de spots qui éclairent chacun à sa manière l'espace sémantique et qui permettent ainsi d'échapper à une conception trop étroite de la sémantique, c'est là leur caractère périphérique. En outre, nous les avons rangés par écart croissant au regard d'une approche étroitement linguistique du sens exprimé dans le discours et plus ou moins fixé dans la langue.

Dans sa contribution, The semantic-pragmatics interface: The role of speaker intentions and the nature of implicit meaning aspects, Kristin Börjesson se concentre sur l'interface entre les deux domaines traditionnellement distincts de la sémantique et de la pragmatique linguistiques. Elle nous brosse l'état actuel de la discussion sur la pertinence de cette distinction méthodologique en partant de l'analyse du philosophe P. Grice. Pour P. Grice, le sens d'une proposition se construit à deux niveaux, d'abord par composition sémantique à partir des significations lexicales (domaine étroit de la sémantique) puis, prenant en compte le contexte étroit, par l'adjonction d'ajustements référentiels (dans un espace d'interface entre sémantique et pragmatique). Le résultat (« ce qui est dit ») constitue la base d'inférences pragmatiques relatives aux implicatures conversationnelles et aux actes de langage, donc à l'intentionnalité de l'énonciateur, révélant « ce qui est pensé », qui relève du domaine étroit de la pragmatique. Ultérieurement, la théorie de la Pertinence a déplacé le curseur en intégrant dans la pragmatique le domaine $\mathrm{d}$ 'interface selon P. Grice et en introduisant la notion d'enrichissement libre (les motivations du choix d'un mode d'expression) dont la prise en compte permet l'explicitation du contenu intentionnel de l'énoncé (son explicature). L'auteur 
défend toutefois l'idée que le processus d'enrichissement libre est fonction du contexte discursif, sans spéculation sur le contenu intentionnel de l'énoncé.

L'article de Steven Schoonjans, Paul Sambre, Geert Brône et Kurt Feyaerts, Vers une analyse multimodale du sens : perspectives constructionnelles sur la gestualité co-grammaticale, porte sur la communication multimodale du sens et questionne donc la place de la sémantique linguistique dans le cadre sémiotique, en fonction des hypothèses de la linguistique cognitive actuelle. Les auteurs proposent d'étendre l'hypothèse cognitiviste d'une représentation conceptuelle schématique fondée sur les constructions grammaticales en y intégrant une grammaire de la communication gestuelle, d'où la notion de " gestualité co-grammaticale ». Leur première analyse de cas porte sur l'emploi pertinent de particules modales de l'allemand. Cet emploi est lié à deux types de gestes, les uns à valeur modale et les autres liés au type d'illocution. La gestuelle (p. ex. hochement de tête, haussement des épaules, pointage référentiel) et le choix de particules comme ja, eben, einfach ou halt covarient de manière complexe, avec des combinaisons préférentielles. La seconde étude de cas porte sur la complémentarité langue-geste pour exprimer une relation d'instrumentation. Plus l'expression linguistique est générique, plus elle tend à se combiner avec une gestuelle d'explicitation. La conclusion des auteurs est que, dans le cadre des Grammaires de construction et d'une linguistique incarnée (théorie de l'enaction), les deux modes d'expression gestuelle et linguistique contribuent conjointement à l'élaboration de «Constructions multimodales ».

Dans leur contribution Sémantique distributionnelle en linguistique de corpus, Ann Bertels et Kris Heylen posent une autre question actuellement jugée essentielle par une partie de la communauté des linguistes, celle (wittgensteinienne) de la détermination du sens à partir des usages, tels qu'ils sont repérables à l'aide d'analyses de corpus textuels, ce qui conduit à l'identification de " motifs » sémantiques. Les modèles sémantiques distributionnels (la méthode de repérage des collocations et colligations de J. Sinclair et celle plus sophistiquée statistiquement du Behavioural Profile) se proposent de calculer la proximité sémantique entre items lexicaux en fonction du partage de contextes dans un corpus donné. Les outils les plus récents d'analyse distributionnelle permettent de procéder automatiquement aussi bien à l'identification d'indices contextuels qu'à la classification d'occurrences, conduisant au repérage de patrons et de motifs. L'article vise à fournir une présentation élémentaire de la modélisation distributionnelle et il discute des applications lexicologiques concernant l'analyse de la polysémie et de la variation lexicale. Les experts humains ont besoin de s'appuyer sur la visualisation des résultats du calcul statistique des structures sémantiques et la dernière partie de l'article est consacrée à cette question, ce qui pose cependant la question de la lisibilité des résultats de l'Analyse Factorielle des Correspondances. 
La contribution de Caleb Everett, Mapping explorations of linguistic influences on nonlinguistic thought, nous renvoie à une question remise à jour par les publications de J. Lucy au début des années 1990, celle de la pertinence de l'hypothèse dite « relativiste » du modelage de notre vision du monde par notre langue maternelle, provenant de W. von Humboldt, transmise par les anthropologues et linguistes F. Boas et E. Sapir et développée par B. L. Whorf. L'auteur montre que les linguistes qui ont mis récemment en évidence l'impact récurrent de disparités interlinguistiques sur la cognition extralinguistique, au lieu de revenir au raisonnement de $\mathrm{B}$. L. Whorf, s'emploient à mieux éclairer le recouvrement entre la cognition linguistique et extralinguistique. Ces études, de type psycho-expérimental, élucident l'influence du langage sur d'autres formes de pensée tout en évitant des caractérisations simplistes de la diversité des structures linguistiques ou des peuples qui parlent les langues concernées. C. Everett énumère les nombreux domaines cognitifs et sémantiques qui ont fait l'objet d'une attention particulière dans les travaux de référence sur cette question, en se concentrant sur les principales voies selon lesquelles on a pu montrer que les traits linguistiques relatifs, en particulier, à trois domaines sémantiques (la numération, l'espace et la désignation des couleurs) correspondent de manière prédictible à des patrons associés de la pensée extralinguistique. Il note cependant que, dans la littérature de vulgarisation, le débat n'arrive pas à s'élever au-dessus d'un ressassement des idées pionnières de B. L. Whorf.

L'article de Thierry Poibeau, Traduire sans comprendre ? La place de la sémantique en traduction automatique, examine l'évolution de la traduction automatique depuis les années 1990, laquelle vise à abandonner le principe, dans la phase d'analyse du texte-source, de la recherche $d^{\prime}$ 'une « interlangue »- une représentation formelle de son contenu conçue comme un prérequis avant de passer à la phase de synthèse du texte-cible - au profit d'une démarche statistique établissant des correspondances régulièrement attestées sans chercher à représenter le contenu partagé par le texte-source et le texte-cible. L'auteur défend l'idée que les statisticiens, qui pressentent une relation d'équivalence contextuelle entre des segments de deux langues qui se correspondent régulièrement dans des corpus bilingues, font déjà implicitement de la sémantique. Cependant, les corpus bilingues se fondent sur l'alignement de textes de langues plus ou moins bien représentées sur Internet et d'une morphologie plus ou moins complexe. Si la traduction entre langues européennes fonctionne relativement bien, surtout quand la langue-cible est l'anglais avec sa morphologie réduite, il n'en est pas de même si, par exemple, l'une des deux langues a une structure agglutinante, et donc une morphologie foisonnante. Et la solution consistant à recourir à l'anglais comme langue-pivot dans la traduction entre deux langues "rares » en format numérique (p. ex. grec $\leftrightarrow$ anglais $\leftrightarrow$ finnois) ne peut pas présenter les qualités de l'interlangue ambitionnée dans les années 1970-1990, car elle charrie des idiotismes de l'anglais qui entraînent des non-sens. En fin de compte, l'approche statistique met en lumière " la nature fondamentalement statistique des langues », mais, au-delà de correspondances lexico-syntaxiques élémentaires, 
elle n'est pas capable de rendre compte d'équivalences entre constructions grammaticales, et l'on peut donc s'attendre à ce que les traductions automatiques à base statistique soient confinées à un seuil de qualité médiocre.

La contribution de Jacques François, Le débat sur la place de la sémantique dans l'acquisition des structures argumentales, vise à tirer les leçons de la discussion engendrée par les publications psycholinguistiques de S. Pinker ${ }^{4}$ et la réplique de M. Tomasello ${ }^{5}$. En 2008, M. Bowerman et W. Croft ont soumis la thèse de S. Pinker à une critique détaillée intégrant les acquis de M. Tomasello pour l'acquisition de l'anglais et ceux du Modèle de Compétition de E. Bates et B. MacWhinney (mesurant la pertinence de différents types d'amorçage dans l'acquisition comme langue-première d'un vaste éventail de langues typologiquement diverses). L'article se concentre sur la question spécifique de l'acquisition des trois types d'alternances syntaxiques (dative, locative et causative) fréquentes dans la grammaire de la proposition en anglais. L'explication de S. Pinker est brillante dans le cadre de l'hypothèse de la Grammaire Universelle, mais celle, constructionnelle et "basée sur l'usage», de M. Bowerman et W. Croft (spécialement pour l'alternance causative) permet de résoudre le «paradoxe de Baker» (la distinction progressive par le jeune locuteur entre des verbes de sens apparenté les uns permettant ces alternances, les autres les excluant) sans recours à cette hypothèse. L'auteur propose finalement d'adopter une approche dynamique et d'intégrer à l'analyse une composante prédictive sur l'évolution de structures instables ( $\mathrm{p}$. ex. Les ventes de la société explosent $\rightarrow$ La société explose ses ventes).

Avec Sémantique et Aphasie: Approche neuropsycholinguistique des processus cognitifs/linguistiques de haut niveau, Jean-Luc Nespoulous nous entraîne dans l'univers oppressant de l'aphasiologie. Méthodologiquement, sa contribution s'appuie sur la comparaison entre deux pathologies A et B du cerveau parlant et, ce faisant, sur les notions de double dissociation (la présence de l'une des deux pathologies A ou B en l'absence de l'autre chez deux patients atteste leur indépendance mutuelle), de dissociation simple (inversement, l'absence d'attestation $\mathrm{d}$ 'une pathologie $\mathrm{B}$ indépendamment de la pathologie $\mathrm{A}$ atteste la dépendance de $\mathrm{B}$ par rapport à $\mathrm{A}$, selon une « hiérarchie de complexité ») et de symptômes " associés » (révélant le chevauchement entre des processus linguistiques et des processus cognitifs plus généraux). Dans le premier cas, l'auteur s'appuie sur les dissociations entre la gestion du " référent » et celle du "signe linguistique » (une agnosie/apraxie dans le premier cas, une aphasie dans le second). Il examine, en particulier, la question de la dissociation entre noms et verbes (aisément confondue avec celle entre représentations d'objet et d'action). Dans le second cas, il s'appuie sur la dissociation "simple » entre la gestion des signes dotés

\footnotetext{
4. En particulier, Learnability and Cognition: The Acquisition of Argument Structure, 1989.

5. En particulier dans First verbs: A case study of early grammatical acquisition (1992), suivi de Constructing a language: A usage base theory of language acquisition (2003).
} 
d'un référent (le vocabulaire concret) et celle des signes qui en sont dénués (le vocabulaire abstrait). Dans le troisième cas, il évoque l'usage excessif qui a été fait de la notion de neurone miroir (à propos du parallélisme entre l'accomplissement, la perception et la description d'une action) et la confiance excessive dans la capacité de la neuro-imagerie fonctionnelle à cerner les localisations cérébrales du langage. Mais, comme l'avait déjà pressenti le neuropsychologue K. Lichtheim en 1885 , cette contribution montre à quel point l'observation des troubles du langage, et particulièrement des aphasies, éclaire l'architecture fonctionnelle du langage et de la cognition humaine.

La dernière contribution est celle de Jean-Louis Dessalles consacrée à L'émergence du sens au cours de l'évolution. Après une première période de « linguistique naturaliste » à la suite de la publication de L'Origine des espèces de C. Darwin (1859), cette thématique a connu une éclipse d'un siècle avant de figurer à nouveau à l'ordre du jour des « biolinguistes » autour de 1990. La question de l'émergence du sens a alors attiré l'attention de chercheurs tels que L. Steels, D. Bickerton, M. Arbib ou J. Hurford. L'auteur favorise la thèse de P. Gärdenfors selon laquelle « les significations lexicales se situent dans les même espaces que les perceptions ». Examinant l'hypothèse du " protolangage " des anciens humains proposée par D. Bickerton, il écarte à la fois l'hypothèse du langage de la pensée (J. Fodor) et celle de l'antériorité de la pensée prédicative sur la pensée communicative (N. Chomsky). J.-L. Dessalles attache une importance décisive à la relation de prédication comme prérequis pour l'opération de négation (et, plus généralement, de contraste) qui représente un saut cognitif puisqu'elle n'est plus en prise sur une perception. Contrairement à l'hypothèse d'une apparition fortuite de la récursivité syntaxique, l'auteur insiste sur la priorité de la récursivité sémantique, sous la forme du partage d'une variable entre deux prédicats (principe de liaison sémantique) comme dans La sœur de ton amie est partie analysable en scur_de (x,y), ami_de (y, allocutaire) et être-parti (x). Selon J.-L. Dessalles, le scénario de l'émergence du sens présente donc trois phases, celle de la combinaison entre des mots correspondant chacun à un type de perception, celle de l'opération de prédication permettant les contrastes et la négation, enfin celle de la combinaison de deux prédicats par le partage d'une variable.

\section{Références}

BALDINGER K. (1984), Vers une sémantique moderne, Paris : Klincksieck.

BARTHES R. (1964), L'Aventure sémiologique, Paris : Le Seuil.

BATES E. \& MACWhinney B. (1989), "Functionalism and the competition model", in

B. MacWhinney \& E. Bates (eds), The Crosslinguistic Study of Sentence Processing, Cambridge: Cambridge University Press, 3-76.

BoAs F. (1911), Handbook of American Indian Languages, Washington: Government Printing Office. 
Bowerman M. \& CROFT W. (2008), “The acquisition of the English Causative Alternation”, in M. Bowerman \& P. Brown (eds), Crosslinguistic Perspectives on Argument Structure. Implications for Learnability, New York/London: Lawrence Erlbaum Associates, 279-308.

BRES J. et al. (éds) (2005), Dialogisme et polyphonie. Approches linguistiques, Bruxelles : De Boeck Supérieur.

Chomsky N. (2002), On Nature and Language, Cambridge: Cambridge University Press.

CoSERIU E. (1958), Sincronia, diacronia e historia, Montevideo : Universidad de la Republica.

COSERIU E. (2001), L'Homme et son langage, Louvain : Peeters.

DARWIN C. (1859), On the Origin of Species, London: John Murray.

DowTY D. (1979), Word Meaning and Montague Grammar, Dordrecht: Reidel.

Ducrot O. (1989), Logique, structure, énonciation. Lectures sur le langage, Paris : Éditions de Minuit.

Everett C. (2016), "Les explorations des influences linguistiques sur la pensée non linguistique ", Langages 201. (ce volume)

Fodor J. A. (1998), Concepts: Where Cognitive Science Went Wrong, Oxford: Clarendon Press.

GÄRDENFORS P. (2014), The Geometry of Meaning - Semantics Based on Conceptual Spaces, Cambridge (MA): The MIT Press.

GeERAERTS D. (2010), Theories of Lexical Semantics, Oxford/New York: Oxford University Press.

Goodenough W. (1956), "Componential analysis und the study of meaning”, Language 32, 195-216.

GreimAs A. J. (1965), Sémantique structurale, Paris : Larousse.

GRICE P. (1989), Studies in the Ways of Words, Cambridge/London: Harvard University Press.

HJelmsLev L. ([1943] 2000), Prolégomènes à une théorie du langage, Paris : Minuit.

HJelmslev L. (1997), Essais linguistiques, Textes édités par F. Rastier, Paris: Presses Universitaires de France.

Humboldt W. (von) (1974), Introduction à l'œuvre sur le Kavi, et autres essais, Paris : Éditions du Seuil.

KAMP H. \& ReYLE U. (1993), From Discourse to Logic, Dordrecht: Kluwer.

LAKoff G. \& Johnson M. (1999), Philosophy in the Flesh: The Embodied Mind and its Challenge to Western Thought, New York: Basic Books.

LANGACKER R. W. (2008), Cognitive Grammar. A Basic Introduction, Oxford: Oxford University Press.

LICHTHEIM K. (1885), “On aphasia”, Brain 7, 433-484.

LOUNDSBURY F. (1964), "The structural analysis of kinship semantics", in H. Lunt (ed.), Proceedings of the Ninth International Congress of Linguists, The Hague: Mouton, 10731090.

Lucy J. A. (1992a), Grammatical Categories and Cognition: A Case Study of the Linguistic Relativity Hypothesis, Cambridge: Cambridge University Press.

LuCY J. A. (1992b), Language Diversity and Thought: A Reformulation of the Linguistic Relativity Hypothesis, Cambridge: Cambridge University Press.

MoEsCHLER J. (1989), Modéliser le dialogue, Paris : Hermès.

SAPIR E. (1970), Le Langage, Paris : Payot.

SinclaIR J. (1991), Corpus, Concordance, Collocation, Oxford: Oxford University Press. 
Pour une vision périphérique de la sémantique linguistique présente et à venir

TRIER J. ([1931] 1973), Der deutsche Wortschatz im Sinnbezirk des Verstandes : von den Anfängen bis zum Beginn des 13. Jahrhunderts, Heidelberg: Winter. [Le Vocabulaire allemand dans le champ conceptuel de l'entendement : des origines au début du XIII siècle, rééd. 1973, Heidelberg : Winter.]

WEISGERBER L. (1929), Muttersprache und Geistesbildung, Göttingen : Vandenhoeck \& Ruprecht. WEISGERBER L. (1962), Die sprachliche Gestaltung der Welt, Düsseldorf : Schwann.

WHORF B. L. (1969), Linguistique et anthropologie, Paris : Denoël/Gonthier.

WierzBicka A. (1972), Semantic Primitives, Francfort/Main: Athenäum.

WitTgensteIn L. (1961), Tractatus logico-philosophicus, suivi de Investigations philosophiques, Paris : Gallimard. 